\title{
Surface Registration by Optimization in Constrained Diffeomorphism Space
}

\author{
Wei Zeng \\ Florida International University, Florida \\ wzeng@cs.fiu.edu \\ Lok Ming Lui \\ Chinese University of Hong Kong, Hong Kong \\ lmlui@math.cuhk.edu.hk \\ Xianfeng $\mathrm{Gu}$ \\ Stony Brook University, New York \\ guecs. stonybrook. edu
}

\begin{abstract}
This work proposes a novel framework for optimization in the constrained diffeomorphism space for deformable surface registration. First the diffeomorphism space is modeled as a special complex functional space on the source surface, the Beltrami coefficient space. The physically plausible constraints, in terms of feature landmarks and deformation types, define subspaces in the Beltrami coefficient space. Then the harmonic energy of the registration is minimized in the constrained subspaces. The minimization is achieved by alternating two steps: 1) optimization - diffuse the Beltrami coefficient, and 2) projection - first deform the conformal structure by the current Beltrami coefficient and then compose with a harmonic map from the deformed conformal structure to the target. The registration result is $d$ iffeomorphic, satisfies the physical landmark and deformation constraints, and minimizes the conformality distortion. Experiments on human facial surfaces demonstrate the efficiency and efficacy of the proposed registration framework.
\end{abstract}

\section{Introduction}

Surface registration plays a fundamental role in computer vision and engineering fields. Given the source and target surfaces with Riemannian metrics, $\left(S_{k}, \mathbf{g}_{k}\right), k=1,2$, the registration problem is to find a mapping $f: S_{1} \rightarrow S_{2}$. In principle, the diffeomorphisms between the two surfaces form an infinite dimensional space. It is intrinsically challenging to find an optimal one in this mapping space. In general, the desirable $f$ should satisfy many criteria as follows:

1. It should be a diffeomorphism, namely smooth and bijective, for surfaces with any topologies.

2. It should minimize distortions. For example, conformal registration minimizes the angle distortion, optimal mass transport map minimizes the area distortion, harmonic map minimizes the elastic membrane energy, and biharmonic map minimizes the thin plate energy.

3. It should satisfy physical feature landmark constraints. Suppose $\left\{p_{i}\right\}$ and $\left\{q_{i}\right\}, i=1 . . m$ are given, $f\left(p_{i}\right)=q_{i}$.

4. It should respect and reflect the physical deformation reality, determined by physical characteristics of materials and governed by physical laws.

5. The computation should be efficient and accurate.

Although there are many existing methods for deformable surface registration, few of them satisfy the above criteria. The iterative closest point (ICP) method [2] can handle surfaces which differ by a rigid motion, but cannot handle nonrigid isometric deformation. Conformal parameterization based registration methods [18] can handle nonrigid isometric deformation, but have difficulties for nonconformal deformation, such as large human expression changes. Optimal mass transportation maps cannot handle landmarks or surfaces with complicated topologies. Harmonic maps may not be diffeomorphic if the target domain has complicated topologies or with landmark constraints. Biharmonic maps cannot handle surfaces with landmarks.

This work addresses the deformable surface registration problem and formulates it as an optimization problem in diffeomorphism space. Basically, any diffeomorphism $f: S_{1} \rightarrow S_{2}$ induces a complex differential $\mu(f)$ on the source, $\|\mu(f)\|_{\infty}<1$, the so-called Beltrami coefficient. Reversely, the diffeormorphism can be fully recovered by its Beltrami coefficient. Therefore, the diffeomorphis$\mathrm{m}$ space is converted to a complex functional space on the source, denoted as $\mathscr{B}\left(S_{1}\right)$. We perform the optimization in this Beltrami coefficient space.

Furthermore, depending on the physical nature of the mappings, we only consider those diffeomorphisms that are physically plausible, whose Beltrami coefficients form subspaces in $\mathscr{C}\left(S_{1}\right)$. In this work, we add two types of physical constraints to the diffeomorphism between human facial surfaces: 1) landmark constraints. Assume the map- 
ping corresponding to a given $\mu$ is $f^{\mu}$, then we require $\left.f^{\mu}\left(p_{i}\right)=q_{i}, i=1 . . m ; 2\right)$ constraints related to the facial skin deformation. The skin in forehead and nose regions, denoted as $\Omega$, has much less deformations during expression change, so we can require the deformation in $\Omega$ to be close to an isometry and its Beltrami coefficient to be zero. Therefore our optimization is performed in the subspace

$$
\mathscr{C}\left(S_{1}\right):=\mathscr{B}\left(S_{1}\right) \bigcap\left\{\mu|\mu|_{\Omega} \equiv 0\right\} \bigcap\left\{\mu \mid f^{\mu}\left(p_{i}\right)=q_{i}, \forall i\right\} .
$$

The energy we try to optimize is the conformality distortion of the mapping, which is achieved by minimizing the harmonic energy of the Beltrami coefficient in the subspace. The computation process has mainly two steps:

1. Optimization: diffuse current Beltrami coefficient $\mu$ to reduce its harmonic energy in the whole space $\mathscr{B}\left(S_{1}\right)$;

2. Projection: project $\mu \in \mathscr{B}\left(S_{1}\right)$ to the constraint subspace $\mathscr{C}\left(S_{1}\right)$.

\subsection{Previous Works}

Surface registration and tracking has a broad range of applications, such as shape matching and recognition for deformable objects in computer vision, shape modeling in geometric modeling, morphological study in medical imaging, and animation in game industry $[6,8,16,17,20]$. In recent years, 3D surface registration methods have been intensively explored. Most existing methods directly deal with nonrigid deformations in $\mathbb{R}^{3}$, but always stop at a local optima and hardly get a global solution.

Surface conformal mapping based methods have been developed for surface matching $[5,15,10]$, registration [3, 22], and tracking [23]. The key idea is to map surfaces to $2 \mathrm{D}$ canonical domains and then solve the surface registration problem as an image registration problem. These methods can handle nonrigid deformations. Surface quasiconformal mapping has great potential to deal with largescale nonrigid deformations. The Beltrami holomorphic flow method [12] and the auxiliary metric method [21] were introduced for computing surface quasiconformal maps and further applications of surface registration, compression and inpainting. Extremal quasiconformal maps between two planar disks with Dirichlet boundary conditions were recently explored in [19] for surface parameterization. Teichmüller map between surfaces with landmark point constraints was recently introduced in [9].

In real applications, landmark constraints are usually introduced to guide surface registration, however, hard constraints sometimes will cause flipping in the mapping (i.e., not a diffeomorphism). The proposed method in this work can guarantee both diffeomorphism and hard landmark constraints. Besides that, we introduce physical deformation constraints for those regions without specific conformality distortions during surface deformation, which are denoted by Beltrami coefficient $\mu$.

\subsection{Contribution}

The major contribution of the current work is to propose a novel registration framework for deformable surfaces with general and large deformations and landmark point constraints. The registration is formulated as an optimization problem in a constrained diffeomorphism space. To our best knowledge, this is the first work which 1) satisfies landmark and physical deformation constraints, 2) guarantees diffeomorphism and 3) has minimal conformality distortion.

The diffeomorphism space is first modeled as a special complex functional space on the source surface, the socalled the Beltrami coefficient space. The landmark constraints and the physically meaningful constraints define the subspaces in the Beltrami coefficient space. Then the harmonic energy of the registration is minimized in the constrained subspaces by alternating two main steps: 1) optimization and 2) projection. The optimization step is to diffuse the Beltrami coefficient; the projection step first deforms the conformal structure by the current Beltrami coefficient, and then composes with a harmonic map from the deformed conformal structure to the target.

\section{Theoretic Background}

This section briefly introduces the theoretic background. We refer readers to [13] for detailed explanations in differential geometry, [14] for harmonic mappings and [1] for quasiconformal mapping.

\subsection{Conformal Mapping}

Given two metric surfaces $\left(S_{1}, \mathbf{g}_{1}\right)$ and $\left(S_{2}, \mathbf{g}_{2}\right)$, a mapping $\phi: S_{1} \rightarrow S_{2}$ is called a conformal mapping or anglepreserving mapping, if the pullback metric induced by $\phi$ on $S_{1}, \phi^{*} \mathbf{g}_{2}=e^{2 \lambda} \mathbf{g}_{1}$, where $\lambda$ is called the conformal factor. Conformal mappings must be diffeomorphisms.

According to surface uniformization theorem, any metric surface can be conformally mapped to $2 \mathrm{D}$ canonical domain. The mapping between two metric surfaces $\left(S_{k}, \mathbf{g}_{k}\right)$ can be converted to the mapping between their conformal (isothermal) domains, as shown in the following diagram:

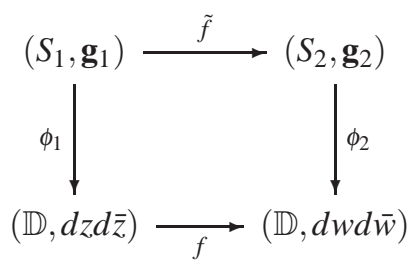

Suppose $z, w$ are conformal parameters of $S_{1}$ and $S_{2}$, respectively, and $\phi_{k}: S_{k} \rightarrow \mathbb{D}$ are the corresponding parameterizations. The mapping between parameter domains $f: \mathbb{D} \rightarrow \mathbb{D}$ induces a mapping $\tilde{f}: S_{1} \rightarrow S_{2}$ between two surfaces. $f$ is a diffeomorphism if and only if $\tilde{f}$ is a diffeomorphism. 


\subsection{Surface Ricci Flow}

An efficient way to compute conformal mapping is Hamilton's surface Ricci flow [7]. Let the metric tensor of the surface is $\mathbf{g}=\left(g_{i j}\right)$. Assume the user prescribes a target curvature $\bar{K}(p)$. Then the Ricci flow is defined as

$$
\frac{\partial g_{i j}(p, t)}{\partial t}=2(\bar{K}(p)-K(p, t)) g_{i j}(p, t) .
$$

Hamilton and Chow [4, 7] proved that when $t \rightarrow \infty, K(\infty)$ converges to the target curvature $\bar{K}$ exponentially fast.

Suppose $(S, \mathbf{g})$ is a topological disk with four boundary points as markers $\left\{v_{0}, v_{1}, v_{2}, v_{3}\right\}$. We can set the target Gaussian curvature $\bar{K}(p)$ to be zero at every interior point, the target geodesic curvature to be zero at every boundary point except the markers, and the target exterior angles at markers to be $\frac{\pi}{2}$. Then Ricci flow conformally deforms the surface to be a planar rectangle. The mapping is denoted as $\phi:(S, \mathbf{g}) \rightarrow(R, d z d \bar{z})$. Furthermore, the four markers are mapped to the four corners, respectively.

\subsection{Quasiconformal Mapping}

Given two surfaces with same topology, in general, there doesn't exist a conformal mapping between them, but there must be quasiconformal (q.c.) mappings between them.

Let complex differential operators be $\partial_{z}=\frac{1}{2}\left(\partial_{x}-\right.$ $\left.i \partial_{y}\right), \partial_{\bar{z}}=\frac{1}{2}\left(\partial_{x}+i \partial_{y}\right)$. A complex function $f: \mathbb{C} \rightarrow \mathbb{C}$ is holomorphic, if $\partial_{\bar{z}} f=0$. The Beltrami coefficient $\mu_{f}$ of the mapping $f: z \rightarrow w(z)$ is defined as

$$
w_{\bar{z}}=\mu_{f}(z) w_{z}
$$

called the Beltrami equation. The mappings between the unit disks and their Beltrami coefficients have one-to-one correspondences. Namely, $\left\{\phi: \mathbb{D} \rightarrow \mathbb{D} \mid J_{\phi}>0\right\} / \operatorname{Mob}(\mathbb{D}) \cong$ $\mathscr{B}(\mathbb{D})$, where $\mathscr{B}(\mathbb{D}):=\left\{\mu: \mathbb{D} \rightarrow \mathbb{C} \mid\|\mu\|_{\infty}<1\right\}$. The left hand side is the space of all diffeomorphisms from the disk to itself, where $J_{\phi}$ is the Jacobian; the right hand side is the functional space of all Beltrami coefficients with norm less than one everywhere. $\operatorname{Mob}(\mathbb{D})$ is Möbius transformation group, each Möbius map has the form $z \rightarrow e^{i \theta} \frac{z-z_{0}}{1-\bar{z}_{0} z}$.

Define the maximal dilation of $f$ as $K_{f}=\frac{1+\|\mu\|_{\infty}}{1-\|\mu\|_{\infty}}$. Intuitively, a q.c. map $f$ maps infinitesimal circles to infinitesimal ellipses, and the ratio between the major axis and the minor axis is given by $K_{f}(z)$. A homeomorphism with dilation less than $K$ is called a $K$-quasiconformal mapping. $f$ is conformal if and only if $\mu_{f}=0$ everywhere and $K_{f}=1$.

\subsection{Auxiliary Metric for Solving Beltrami Equation}

Suppose $f: z \rightarrow w$ is a quasiconformal mapping with Beltrami coefficient $\mu$. The Beltrami equation $w_{\bar{z}}=\mu(z) w_{z}$ can be solved using Ricci flow method as well. Suppose the mapping $f$ maps a topological quadrilateral $S \subset \mathbb{C}$ to a rectangle, and the original Riemannian metric is $\mathbf{g}=d z d \bar{z}$.
We construct an auxiliary metric $\tilde{\mathbf{g}}=|d z+\mu d \bar{z}|^{2}$. Then we use Ricci flow to map $(S, \tilde{\mathbf{g}})$ to a planar rectangle, and the mapping is the quasiconformal map $f$.

\subsection{Harmonic Map}

Let $f:\left(\mathbb{D},|d z|^{2}\right) \rightarrow\left(\mathbb{D},|d w|^{2}\right)$ be a Lipschitz map between two disks, $z=x+i y$ and $w=u+i v$ are complex parameters. The harmonic energy of the map is defined as

$$
E(f)=\int_{\mathbb{D}}\left(\left|w_{z}\right|^{2}+\left|w_{\bar{z}}\right|^{2}\right) d x d y .
$$

Definition 2.1 (Harmonic Map) A critical point of the harmonic energy is called a harmonic map.

The Hopf differential of a mapping $f \phi(f)$ is defined as

$$
\Phi(f)=w_{z} \overline{w_{\bar{z}}} d z^{2} .
$$

If the mapping is harmonic, then it satisfies the Laplace equation $w_{z \bar{z}}=0$. This is equivalent to $\Phi(f)$ being holomorphic. Furthermore, $\Phi(f)$ is zero if and only if $f$ is a conformal mapping. In general, harmonic mapping is unnecessarily diffeomorphic.

Suppose one change the parameterization of the source from $z$ to $\zeta$, such that $\zeta_{\bar{z}}=(\mu+\varepsilon v) \zeta_{z}$, then compute the harmonic map with respect to $\zeta$, denoted as $h^{\mu+\varepsilon v}$, then the variation of the harmonic energy is given by

$$
\frac{d}{d \varepsilon} E\left(h^{\mu+\varepsilon v}\right)=-4 \Re \mathfrak{R} \int_{\mathbb{D}} \Phi\left(h^{\mu}\right) v d \zeta_{x} d \zeta_{y} .
$$

\section{Optimization in Constrained Diffeomor- phism Space}

In our current work, two metric surfaces $\left(S_{k}, \mathbf{g}_{k}\right), k=1,2$ are given with landmarks $\left\{p_{i}\right\} \subset S_{1},\left\{q_{i}\right\} \subset S_{2}, i=1 . . m$ and subregions $\Omega \subset S_{1}$. We aims at finding a map $\tilde{f}:\left(S_{1}, \mathbf{g}_{1}\right) \rightarrow$ $\left(S_{2}, \mathbf{g}_{2}\right)$, such that $\tilde{f}$ is a smooth diffeomorphism and satisfies the landmark constraints, $\tilde{f}\left(p_{i}\right)=q_{i}$, furthermore, $\tilde{f}$ on $\Omega$ is conformal, i.e., $\left.\mu(\tilde{f})\right|_{\Omega} \equiv 0$. Namely, $\mu(f) \in \mathscr{C}\left(S_{1}\right)$, which is the subspace of $\mathscr{B}\left(S_{1}\right)$ defined in (1).

Let $\phi_{k}:\left(S_{k}, \mathbf{g}_{k}\right) \rightarrow R_{k}$ be the conformal parameterization, where $R_{k}$ 's are planar rectangle domains. Let $f: R_{1} \rightarrow R_{2}$ be the corresponding mapping between parameter domains. According to (2), the desired map is $\tilde{f}=\phi_{2}^{-2} \circ f \circ \phi_{1}$. Then the problem is converted to find the optimal diffeomorphism $f$. For convenience, we define two subspaces of $\mathscr{B}\left(R_{1}\right)$,

$$
\begin{aligned}
\mathscr{S}_{0}:=\left\{\mu|\mu|_{\phi_{1}(\Omega)} \equiv 0\right\}, \\
\mathscr{S}_{1}:=\left\{\mu \mid f^{\mu}\left(\phi_{1}\left(p_{i}\right)\right)=\phi_{2}\left(q_{i}\right), \forall i\right\},
\end{aligned}
$$

and formulate our optimization problem as follows:

Problem 3.1 (Constrained Optimal Diffeomorphism)

$$
\mu^{*}=\arg \min _{\mu} \int_{R_{1}}\left(\left|\mu_{z}\right|^{2}+\left|\mu_{\bar{z}}\right|^{2}\right) d x d y,
$$

subject to: 


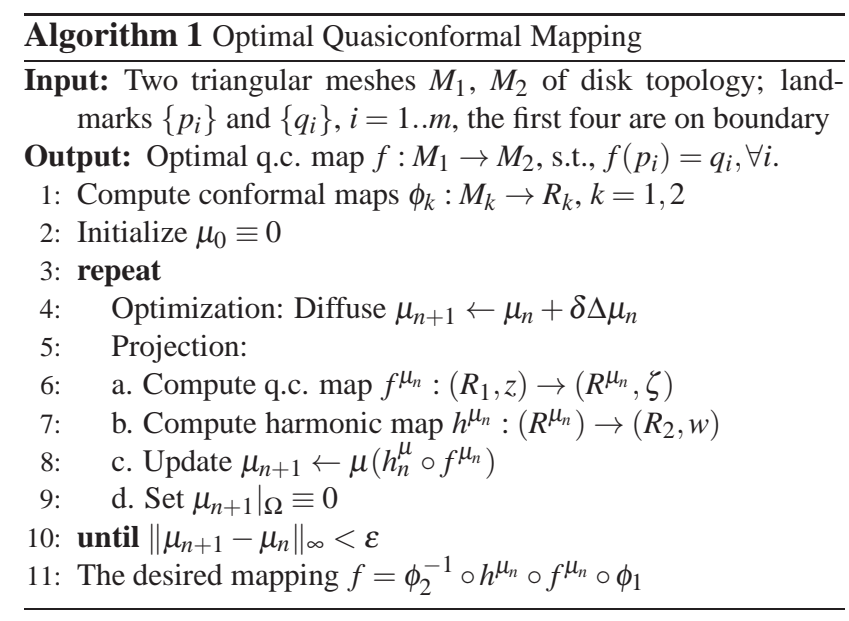

1. diffeomorphism $\|\mu\|_{\infty}<1$,

2. landmark constraints, $f^{\mu}\left(\phi_{1}\left(p_{i}\right)\right)=\phi_{2}\left(q_{i}\right), \forall i$,

3. conformality constraints, $\left.\mu\right|_{\phi_{1}(\Omega)} \equiv 0$,

namely, $\mu^{*} \in \mathscr{B}\left(R_{1}\right) \cap \mathscr{S}_{0} \cap \mathscr{S}_{1}$.

The optimization is performed by alternating two main steps, Optimization and Projection.

Optimization The optimization of $\mu$ is straightforward. We apply the traditional heat flow method,

$$
\frac{\partial \mu}{\partial t}=-\mu_{z \bar{z}}
$$

which will reduce the harmonic energy of $\mu$.

Projection First, we project $\mu$ to the subspace $\mathscr{B}\left(R_{1}\right) \cap$ $\mathscr{S}_{0}$. This can be easily achieved by directly setting $\mu$ to be zero in the region $\phi_{1}(\Omega)$.

Then, we project the current $\mu$ to the subspace $\mathscr{B}\left(R_{1}\right) \cap$ $\mathscr{S}_{1}$. We propose to solve the problem by two stages:

$$
\left(R_{1}, z\right) \stackrel{f^{\mu}}{\longrightarrow}\left(R^{\mu}, \zeta\right) \stackrel{h^{\mu}}{\longrightarrow}\left(R_{2}, w\right)
$$

Stage 1: For a given $\mu$ on $R_{1}$, by solving Beltrami equation, we map $R_{1}$ to an intermediate parameter domain $R^{\mu}$ with complex parameter $\zeta, f^{\mu}:\left(R_{1}, z\right) \rightarrow\left(R^{\mu}, \zeta\right),\left.\mu\right|_{\Omega} \equiv 0$.

Stage 2: We compute a harmonic map from the intermediate domain to the target domain satisfying the landmark constraints, $h^{\mu}:\left(R^{\mu}, \zeta\right) \rightarrow\left(R_{2}, w\right)$. The composition map is $h^{\mu} \circ f^{\mu}$, whose Beltrami coefficient is in the subspace $\mathscr{B}\left(R_{1}\right) \cap \mathscr{S}_{1}$. We replace the current $\mu$ by $\mu\left(h^{\mu} \circ f^{\mu}\right)$.

The computational pipeline is shown in Algorithm 1. Note that, after the second step of the projection to $\mathscr{S}_{1}$, the Beltrami coefficient may be outside $\mathscr{S}_{0}$. We can alternate the two projection steps several times to ensure the final $\mu$ is inside $\mathscr{S}_{0} \cap \mathscr{S}_{1}$. The algorithm is guaranteed to converge to the unique extremal quasiconformal map; the proof is using the method in [11].

\section{Computational Algorithms}

This section explains the computational algorithms in detail. The facial surfaces are genus zero and with a single boundary. They are represented as triangle meshes, denoted as $M=(V, E, F)$, where $V, E, F$ represent vertex, edge and face sets, respectively. Suppose four boundary vertices are labeled as the markers, $\left\{v_{0}, v_{1}, v_{2}, v_{3}\right\} \subset \partial M$.

\subsection{Harmonic Mapping}

The discrete harmonic mapping can be computed by solving the Dirichlet problem. The cotangent edge weight is defined as follows:

$$
w_{i j}=\left\{\begin{array}{ll}
\cot \theta_{i j}^{k}+\cot \theta_{j i}^{l} & {\left[v_{i}, v_{j}\right] \notin \partial M} \\
\cot \theta_{i j}^{k} & {\left[v_{i}, v_{j}\right] \in \partial M}
\end{array},\right.
$$

where $\theta_{i j}^{k}$ is the corner angle in $\left[v_{i}, v_{j}, v_{k}\right]$ at $v_{k}$. Suppose $f: V \rightarrow \mathbb{R}$ is a piecewise linear function, then its harmonic energy is

$$
E(u)=\frac{1}{2} \sum_{\left[v_{i}, v_{j}\right] \in E} w_{i j}\left(f\left(v_{i}\right)-f\left(v_{j}\right)\right)^{2} .
$$

Then the discrete Laplace-Beltrami operator is defined as

$$
\Delta f\left(v_{i}\right)=\sum_{\left[v_{i}, v_{j}\right] \in E} w_{i j}\left(f\left(v_{j}\right)-f\left(v_{i}\right)\right) .
$$

The discrete harmonic map $h: M_{1}-\left\{p_{i}\right\} \rightarrow M_{2}-\left\{q_{i}\right\}$ can be computed by solving the linear system

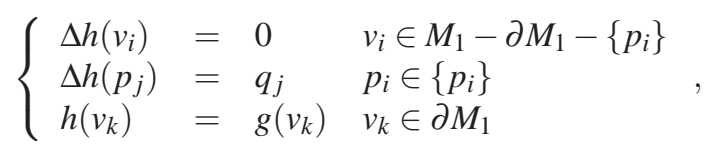

where $g: \partial M_{1} \rightarrow \partial M_{2}$ is a given mapping.

In order to compute the harmonic map with $h^{\mu}$ : $\left(R^{\mu}, \zeta\right) \rightarrow\left(R_{2}, w\right)$, what we need is to modify the cotangent edge weight. Suppose the Beltrami coefficient $\mu: V \rightarrow \mathbb{C}$ is defined on vertices, and $\left[v_{i}, v_{j}, v_{k}\right]$ is isometrically embedded onto the complex plane, with coordinates $z_{i}, z_{j}, z_{k}$. Then we define a linear mapping

$$
\tau(z)=z+\frac{1}{3}\left(\mu\left(v_{i}\right)+\mu\left(v_{j}\right)+\mu\left(v_{k}\right)\right) \bar{z}
$$

then $\tau$ distorts the triangle. We compute the corner angles $\theta_{i}^{j k}(\mu), \theta_{j}^{k i}(\mu), \theta_{k}^{i j}(\mu)$ on the image of $\tau$, compute the cotangent edge weight $w_{i j}(\mu)$ using the distorted angles.

\subsection{Discrete Yamabe Flow}

Discrete Yamabe flow is one scheme for discrete surface Ricci flow. Let $\left[v_{i}, v_{j}\right] \in E$ be an edge with Euclidean length $\beta_{i j}$. Define discrete conformal factor function $u: V \rightarrow \mathbb{R}$, then the discrete conformal deformation of edge length is 
given by $l_{i j} \leftarrow e^{u_{i}} \beta_{i j} e^{u_{j}}$. The discrete vertex curvature $K\left(v_{i}\right)$ is defined as angle deficit

$$
K\left(v_{i}\right)=\left\{\begin{array}{ll}
2 \pi-\sum_{\left[v_{i}, v_{j}, v_{k}\right] \in F} \theta_{i}^{j k} & v_{i} \notin \partial M \\
\pi-\sum_{\left[v_{i}, v_{j}, v_{k}\right] \in F} \theta_{i}^{j k} & v_{i} \in \partial M
\end{array} .\right.
$$

The discrete surface Yamabe flow is defined as

$$
\frac{d u\left(v_{i}\right)}{d t}=\bar{K}\left(v_{i}\right)-K\left(v_{i}\right)
$$

which is the gradient flow of the discrete Yamabe energy,

$$
E(\mathbf{u})=\int_{\mathbf{0}}^{\mathbf{u}} \sum_{i=1}^{|V|}\left(\bar{K}\left(v_{i}\right)-K\left(v_{i}\right)\right) d u_{i},
$$

where $\mathbf{u}=\left(u_{1}, u_{2}, \cdots, u_{|V|}\right)$. Discrete Yamabe energy is convex, the unique global optimum is the desired discrete metric. We can use the Newton's method to optimize it directly, which requires the Hessian matrix. The Hessian matrix is just the conventional cotangent weight LaplaceBeltrami operator in Eqn. (7).

\subsection{Solving Beltrami Equation}

Suppose we want to compute the quasiconformal mapping $f^{\mu}:\left(R_{1}, z\right) \rightarrow\left(R^{\mu}, \zeta\right)$ by solving the Beltrami equation $\zeta_{\bar{z}}=\mu \zeta_{z}$. By the auxiliary metric strategy in Section 2.4 , the quasiconformal map is converted to a conformal mapping. Suppose $\mu$ is defined on each vertex. We define the initial length of an edge $\left[v_{i}, v_{j}\right]$ as

$$
\beta_{i j}^{\mu} \leftarrow\left|\left(z_{j}-z_{i}\right)+\frac{1}{2}\left(\mu\left(v_{i}\right)+\mu\left(v_{j}\right)\right)\left(\bar{z}_{j}-\bar{z}_{i}\right)\right| .
$$

We then compute the conformal map under the new metric using the traditional surface Yamabe flow algorithm [21].

\subsection{Diffusion of Beltrami Coefficient}

First, we compute the cotangent edge weight $w_{i j}$, then form the discrete Laplace-Beltrami operator. The diffusion of Beltrami coefficient is similar to the heat diffusion,

$$
\mu\left(v_{i}\right) \leftarrow \mu\left(v_{i}\right)+\delta \Delta \mu\left(v_{i}\right),
$$

where $\delta>0$ is a small positive constant. The whole algorithm pipeline can be found in Algorithm 1. In practice, the algorithm stably converges for all the experiments.

\section{Experimental Results}

The primary goal of this work is to solve a globally optimal registration problem for surfaces with same topology and general deformations, which is required to be a diffeomorphism and handle physically meaningful constraints, in order to enlarge the scope in practice. The constraints include two forms: 1) feature landmarks and 2) conformality distortion, denoted by Beltrami coefficient $\mu$. Meaningful landmarks can be extracted from texture and/or geometry. Different materials may have different intrinsic elastic properties and different conformality distortions in physical deformations. In this work, we restrict the $\mu$ to be zero on the regions whose deformation is close to isometry, such as the forehead and nose areas during facial expression change.

In the following, we arrange our experiments with three categories of constraints to evaluate the proposed algorithms: 1) Only landmark constraints, 2) Only $\mu$ constraints, and 3) Landmark- $\mu$ constraints. The experimental result$\mathrm{s}$ demonstrate that our method is efficient and effective to register surfaces with large deformations.

\subsection{Optimization with Landmark Constraints}

In this case, we take two surfaces with 16 landmark constraints as input (see Fig. 1 (a)). The deformation of the source surface to the target surface is mainly defined by the landmark correspondence constraints. We first conformally map them to the planar rectangle domain. By a harmonic mapping on the rectangle domain, we get the initial Beltrami coefficients $\mu$ (b). We then apply the iterative optimization process in Algorithm 1 to update $\mu$ and compute the harmonic mapping using the auxiliary metric. The magnitude of Beltrami coefficients $|\mu|$ becomes evener and evener from the initial distribution, and eventually it reaches a steady point that corresponds to the optimal Beltrami differential (c). The algorithm converges for all the experiments. The distribution of $|\mu|$ is evaluated by both the numerical histogram and the visual color-encoded mapping on 3D surface. The resulting mapping is a diffeomorphism and globally unique and optimal in diffeomorphism space.

\subsection{Optimization with $\mu$ Constraints}

Suppose the Beltrami coefficient $\mu$ of the mapping from the source surface to the target has been given already. We can generate the registration directly by the quasiconformal mapping based on the auxiliary metric method [21]. The mapping gives a guidance in diffeomorphism space to reach the optimal one. We then apply the optimization process in Algorithm 1 with $\mu$ constraints to generate a unique quasiconformal mapping with the minimal maximal $|\mu|$.

\subsection{Optimization with Landmark- $\mu$ Constraints}

In practice, not like the explicit feature landmarks, the Beltrami coefficients $\mu$ of encoding quasiconformal deformation is not accessible; usually, it is estimated by first constructing a mapping between two surfaces. If the deformation is conformal (rigid or isometric), then the $\mu$ is zero. Based on this fact, we design our registration strategy by considering physics of specific object deformation and fixing the $\mu$ for specific areas in the registration. 


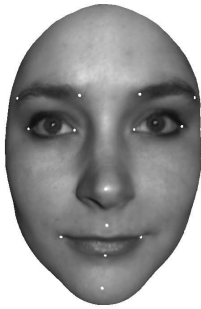

source $S_{1}$

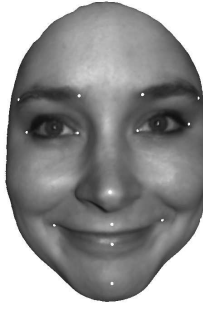

target $S_{2}$

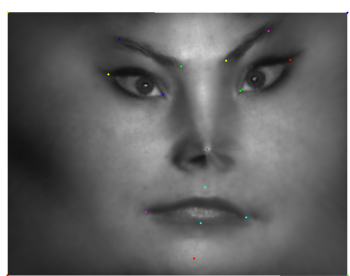

$\phi_{1}: S_{1} \rightarrow R_{1}$

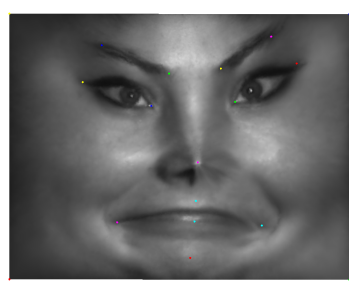

$\phi_{2}: S_{2} \rightarrow R_{2}$
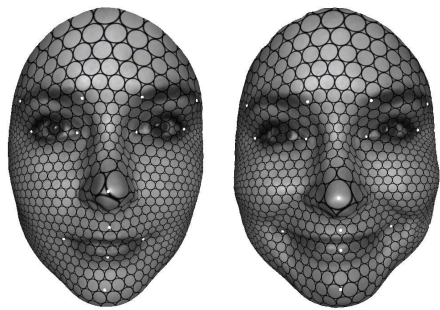

texture map by $\phi_{1}$ texture map by $\phi_{2}$

(a) conformal mappings

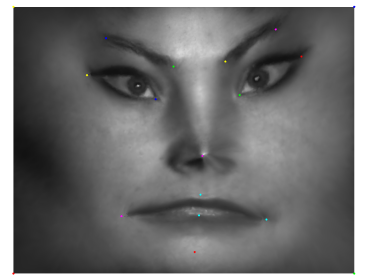

$f_{L}^{0}: R_{1} \rightarrow R_{2}$

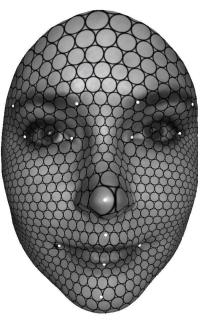

$\tilde{f}_{L}^{0}: S_{1} \rightarrow S_{2}$

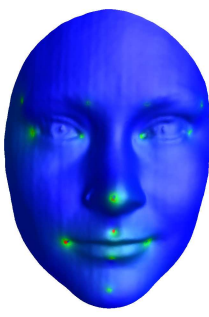

$\left|\mu\left(\tilde{f}_{L}^{0}\right)\right|_{S_{1}}$

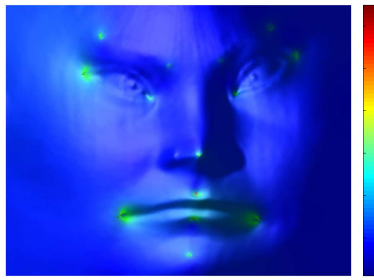

$\left|\mu\left(f_{L}^{0}\right)\right|_{R_{1}}$

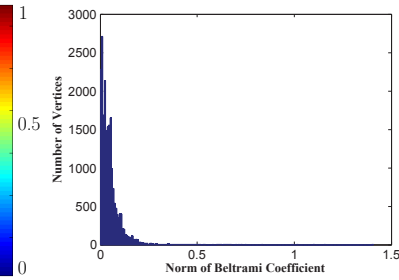

$|\mu|_{\max }=1.407$

(b) initial registration with only landmark constraints

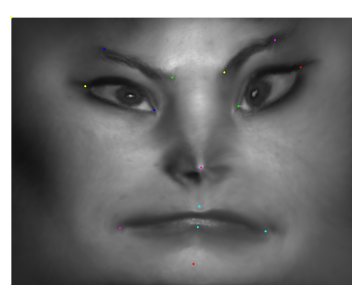

$f_{L}: R_{1} \rightarrow R_{2}$

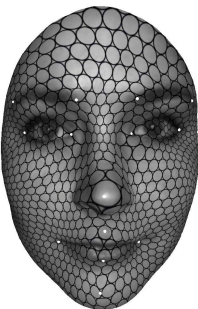

$\tilde{f}_{L}: S_{1} \rightarrow S_{2}$

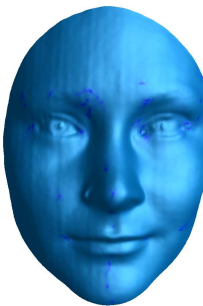

$\left|\mu\left(\tilde{f}_{L}\right)\right|_{S_{1}}$

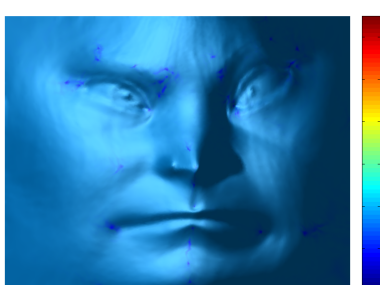

$\left|\mu\left(f_{L}\right)\right|_{R_{1}}$

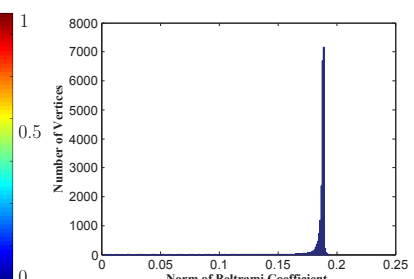

$|\mu|_{\max }=0.221$

(c) optimal registration with only landmark constraints

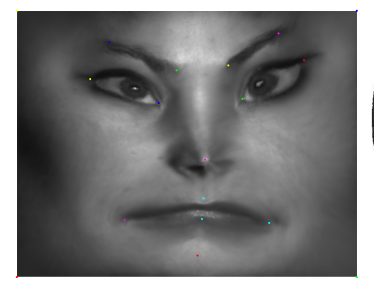

$f_{L-\mu}: R_{1} \rightarrow R_{2}$

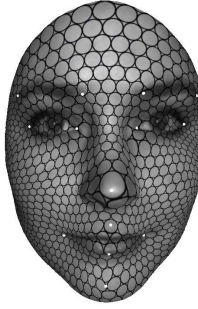

$\tilde{f}_{L-\mu}: S_{1} \rightarrow S_{2}$

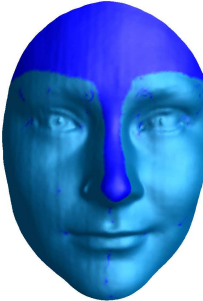

$\left|\mu\left(\tilde{f}_{L-\mu}\right)\right|_{S_{1}}$

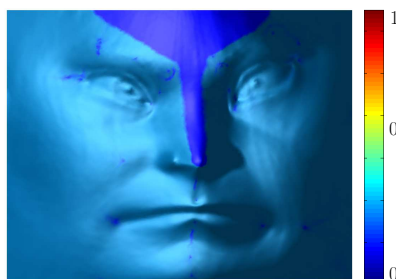

$\left|\mu\left(f_{L-\mu}\right)\right|_{R_{1}}$

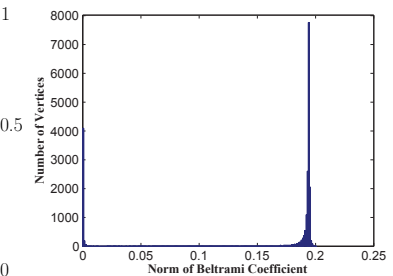

$|\mu|_{\max }=0.242$

(d) optimal registration with landmark- $\mu$ constraints

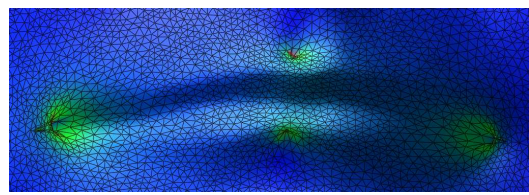

$f_{L}^{0}$

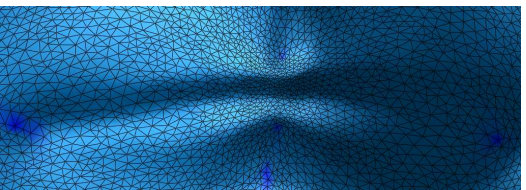

$f_{L}$

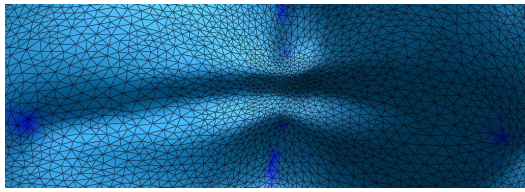

$f_{L-\mu}$

(e) meshing structure at mouth area

Figure 1. Optimization in diffeomorphism space with landmark and/or Beltrami coefficient $(\mu)$ constraints.

We perform testing on human facial surfaces from the same subject, deforming slowly from neutral expression to smile expression (see Fig. 1), where the forehead and nose areas almost have no deformation during expression change. Then we have the following experiments. We set $\mu$ to be zeros for the forehead and nose areas. Then we run the optimization process along with the landmark constraints.
During the update process, we keep $m u$ to be zeros for the constrained areas. Finally we get the optimal registration which respects the realistic physical deformation.

Figure 1 (d) shows the registration with a combination of landmark and $\mu$ constraints. We use circle-packing texture map result on 3D surface to visualize the quasiconformality. It is obvious that on the forehead and nose areas, the 
circles are mapped to circles in the landmark- $\mu$ constrained result (d), while the circles are mapped to ellipses in the only landmark constrained result (c). The distribution of $|\mu|$ is shown by both the histogram and the color-encoded maps on 3D surface and 2D domain, where the blue color denotes zero local stretch, i.e., conformal deformation.

In this registration strategy, the landmark constraints and the $\mu$ together guide the deformation. Note that the landmarks won't stay in $\mu$-constrained areas, since landmark correspondence constraints generally generate quasiconformal deformation. This registration is highly desirable for simulating and recovering the real physical mapping between two frames of one deforming object.

Diffeomorphism Guarantee Figure 1 (e) shows the evolution of meshing structure during the optimization. By harmonic mapping, we can get the initial registration. It is obvious that there is flipping $(|\mu|>1)$ around landmark constrained area (e.g., two mouth corners); the resulting mapping is not a diffeomorphism, which can also be observed in the histogram of $|\mu|$ in (b) $\left(|\mu|_{\max }>1\right)$. By the relaxation process during optimization, the flips are smoothen out gradually and reach a diffeomorphism eventually (c-d).

Registration Accuracy Improvement We evaluate registration accuracy in two ways. Visually, we check the consistency of the circle-packing texture mappings on both source and target surfaces, through the registration mapping. The more consistent the textures on the source and target are, the more accurate the registration is. Observing texture mappings on source in (b-d) and comparing them with the texture mapping on target in (a) (for example, the right inner eye corner area), we can conclude that the optimization improves the registration accuracy. Numerically, with the diffeomorphic mapping, we compute both the texture and geometry distances [22] to evaluate the registration accuracy, $\left(d_{g}, d_{t}\right)=(0.003,0.041)(\mathrm{b}),(0.002,0.034)$ (c), and $(0.001,0.018)(\mathrm{d})$. Thus the registration considering physical deformation constraints is the most accurate one.

Geometric Registration of General Surfaces Besides the physical registration of different expression surfaces from the same subject, the proposed method is also practical to register general surfaces, such as the facial surfaces from different subjects. Figure 2 shows one example of the registration to another target $S_{2}$ from the same source $S_{1}$ in Fig. 1. The geometric distance of the registration is 0.003 , with about $60 \%$ improvement from the initial registration. Note that for this registration case, it is unnecessary to prescribe the $\mu$ constraints, since there is no real physical relation between human faces from different subjects.

\subsection{Discussion}

1) Efficiency - The algorithm has two parts: 1) conformal maps using Yamabe flow, which is a convex optimization by
Newton's method and has time complexity $O(n \log \varepsilon)$ (vertex number $n$, error tolerance $\varepsilon$ ); and 2) harmonic map also using conjugate gradient method, which is $O(n)$. Both of them are robust to geometric noises.

We have tested our algorithms thoroughly on 1500 individual facial surfaces from multiple people's expression sequences. For a surface with $30 k$ triangles, it costs about 10 seconds. The optimization is efficient and practical, and stably converges for all the experiments.

2) Generality - Ricci flow can unify any surface to one of three canonical spaces; any diffeomorphism can be represented by a unique $\mu$, and vice versa. Therefore, the proposed registration framework can be generalized to any types of topology and handle any types of deformations.

3) Rigor - The framework of optimization in diffeomorphism space has solid theoretical background and guarantees the optimal diffeomorphism which exists and is unique.

4) Robustness - The proposed framework can handle a large amount of hard landmark constraints and multiple $\mu$ constrained areas. The algorithm fails when the input feature constraints or surface geometries are inconsistent, such as one surface occluded with a large portion missing.

5) Practicality - In most vision and medical imaging problems, landmarks are associated naturally, such as faces, brains, and other organs with natural deformations. The proposed method guarantees optimal diffeomorphism, and is easy to carry out and compute. It has great potential for real applications surrounding shape matching, tracking, comparison, recognition, and analysis. This proposed method can register surfaces without any constraint as well; with physical constraints, our method can perform better.

6) Comparison - Most existing conformal map based methods cannot guarantee diffeomorphism under hard landmark constraints $[18,23]$ and reach the optimal conformality distortion. The work in [23] only gives correspondence between sparse nets and the correspondence between areas not on the net are completely ignored. Our method gives dense registration over the whole surface. Furthermore, to our best knowledge, this is the first work to introduce physical constraints $\mu$ in registration.

\section{Conclusion}

In this work, we present a novel surface registration framework by optimization in the constrained diffeomorphism space. The landmark and deformation constraints (denoted by Beltrami coefficient $\mu$ ) define the subspaces in the diffeomorphism space. This registration framework guarantees diffeomorphism, satisfies physically meaningful constraints, and minimizes the conformality distortion (the optimal $\mu$ ). It has solid theoretical background and can be generalized to surfaces of any topological types. It is practical and has great potential in solving realistic surface registration problems in engineering and medical fields. 


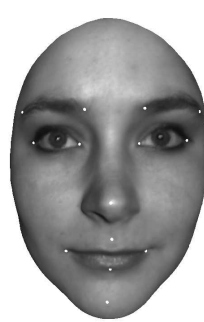

source $S_{1}$

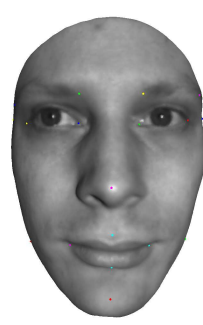

target $S_{2}$

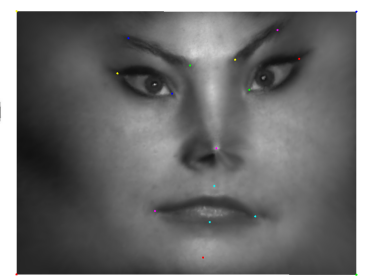

$\phi_{1}: S_{1} \rightarrow R_{1}$

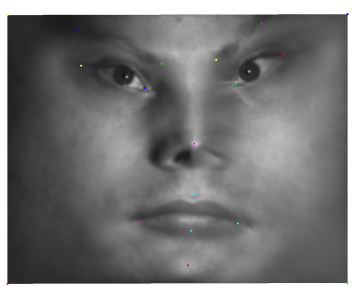

$\phi_{2}: S_{2} \rightarrow R_{2}$

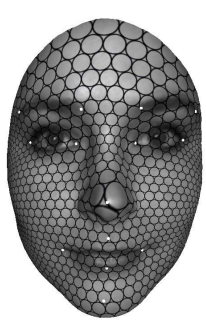

texture map by $\phi_{1}$ texture map by $\phi_{2}$

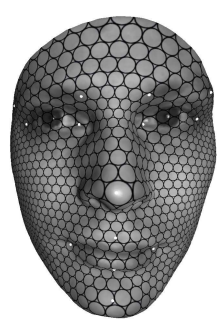

(a) conformal mappings

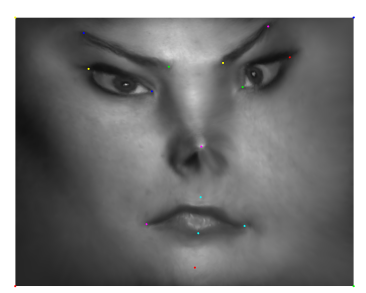

$f_{L}: R_{1} \rightarrow R_{2}$

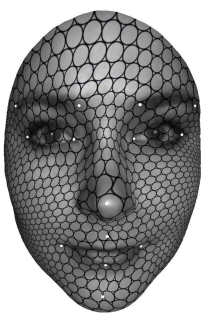

$\tilde{f}_{L}: S_{1} \rightarrow S_{2}$

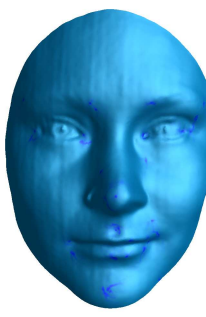

$\left|\mu\left(\tilde{f}_{L}\right)\right|_{S_{1}}$

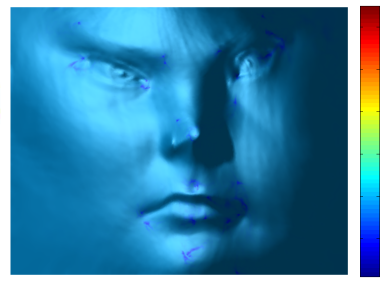

$\left|\mu\left(f_{L}\right)\right|_{R_{1}}$

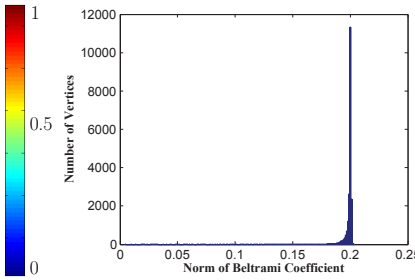

$|\mu|_{\max }=0.222$

(b) optimal registration with only landmark constraints

Figure 2. Geometric registration of human facial surfaces from different subjects.

\section{Acknowledgment}

Xianfeng Gu is supported by NSF DMS-1221339 and Nets-1016829 and AFOSR-FA9550-10-1-0294. Lok Ming Lui is supported by RGC GRF-404612.

\section{References}

[1] L. Ahlfors. Lectures in Quasiconformal Mappings. Van Nostrand Reinhold, New York, 1966. 2

[2] P. J. Besl and N. D. McKay. A method for registration of 3-D shapes. IEEE Trans. Pattern Anal. Mach. Intell., 14(2):239256, 1992. 1

[3] A. M. Bronstein, M. M. Bronstein, and R. Kimmel. Generalized multidimensional scaling: A framework for isometryinvariant partial surface matching. In Proceedings of National Academy of Science, pages 1168-1172, 2006. 2

[4] B. Chow. The ricci flow on the 2-sphere. J. Differential Geom., 33(2):325-334, 1991. 3

[5] M. Feiszli and D. Mumford. Shape representation via conformal mapping. In Proc. SPIE 6498, Computational Imaging $V, 2007.2$

[6] T. Funkhouser, P. Min, M. Kazhdan, J. Chen, A. Halderman, D. Dobkin, and D. Jacobs. A search engine for $3 \mathrm{~d}$ models. ACM TOG, 22(1):83-105, 2003. 2

[7] R. Hamilton. The ricci flow on surfaces. Mathematics and General Relativity, 71:237-262, 1988. 3

[8] D. Huber, A. Kapuria, R. Donamukkala, and M. Hebert. Parts-based 3d object classification. CVPR04, II:82-89. 2

[9] K. Lam, X. Gu, S. Yau, and L. Lui. Teichmuller mapping (tmap) and its applications to landmark matching registrations. SIAM Journal on Imaging Sciences, 2013. 2

[10] Y. Lipman. Conformal Wasserstein distances: Comparing surfaces in polynomial time. In arXiv:1103.4408, 2011. 2

[11] L. M. Lui, X. Gu, and S.-T. Yau. Convergence of an iterative algorithm for teichmüller maps via har- monic energy optimization. Technical report, 2013. ftp://ftp.math.ucla.edu/pub/camreport/cam13-36.pdf. 4

[12] L. M. Lui, T. W. Wong, W. Zeng, X. Gu, P. M. Thompson, T. F. Chan, and S.-T. Yau. Optimization of surface registrations using beltrami holomorphic flow. Journal of Scientific Computing (JSC), 50(3):557-585, 2012. 2

[13] R. Schoen and S.-T. Yau. Lectures on Differential Geometry. International Press of Boston, 1994. 2

[14] R. Schoen and S.-T. Yau. Lecture on Harmonic Maps, volume 2. International Press Incorporated, Boston, 1997. 2

[15] E. Sharon and D. Mumford. 2D-shape analysis using conformal mapping. IJCV, 70:55-75, October 2006. 2

[16] J. Starck and A. Hilton. Correspondence labelling for widetimeframe free-form surface matching. In ICCV, 2007. 2

[17] Y. Sun and M. Abidi. Surface matching by 3d point's fingerprint. ICCV01, II:263-269, 2001. 2

[18] S. Wang, Y. Wang, M. Jin, X. Gu, and D. Samaras. 3D surface matching and recognition using conformal geometry. In CVPRO6, number 2, pages 2453-2460, 2006. 1, 7

[19] O. Weber, A. Myles, and D. Zorin. Computing extremal quasiconformal maps. Comp. Geom. Forum, 31(5):1679-1689, 2012. 2

[20] J. Wyngaerd, L. Gool, R. Koch, and M. Proesmans. Invariant-based registration of surface patches. ICCV99, I:301-306, 1999. 2

[21] W. Zeng and X. Gu. Registration for 3D surfaces with large deformations using quasi-conformal curvature flow. In IEEE CVPR, 2011. 2, 5

[22] W. Zeng, D. Samaras, and X. D. Gu. Ricci flow for 3D shape analysis. IEEE TPAMI, 32(4):662-677, 2010. 2, 7

[23] Y. Zeng, C. Wang, Y. Wang, X. Gu, D. Samaras, and N. Paragios. Dense non-rigid surface registration using high-order graph matching. In IEEE CVPR, 2010. 2, 7 